\title{
Erratum to: Expression of Urokinase-Type Plasminogen Activator and Its Receptor in Gastric Fibroblasts and Effects of Nonsteroidal Antiinflammatory Drugs and Prostaglandin
}

Junichi Iwamoto $\cdot$ Kimiko Takahashi $\cdot$ Yuji Mizokami $\cdot$ Toshiya Otsubo •

Syuuhei Miura · Toshiaki Narasaka · Hiroki Takeyama - Takayuki Omata •

Kouichi Shimokoube · Takeshi Matsuoka

Published online: 8 December 2011

(C) Springer Science+Business Media, LLC 2011

Erratum to: Dig Dis Sci (2003) 48(12):2247-2256

DOI 10.1023/B:DDAS.0000007859.10680.b1

The Corresponding author's first name had been published as Jun-Ichi. The correct version is Junichi Iwamoto and not Jun-Ichi Iwamoto as published.

The online version of the original article can be found under doi:10.1023/B:DDAS.0000007859.10680.b1.

J. Iwamoto $(\varangle) \cdot$ K. Takahashi · Y. Mizokami · T. Otsubo ·

S. Miura $\cdot$ T. Narasaka $\cdot$ H. Takeyama $\cdot$ T. Omata $\cdot$

K. Shimokoube $\cdot$ T. Matsuoka

Fifth Department of Internal Medicine, Tokyo Medical

University, Chuo 3-20-1, Ami, Inashiki, Ibaraki 300 0395, Japan

e-mail: junnki@dg.mbn.or.jp 